\title{
AVALIAÇÃO DA FRAGILIDADE DE IDOSOS INTERNADOS EM SERVIÇO DE EMERGÊNCIA DE UM HOSPITAL UNIVERSITÁRIO*
}

Juliane de Fátima Santos Antunes', Meiry Fernanda Pinto Okuno², Maria Carolina Barbosa Teixeira Lopes³, Cássia Regina Vancini Campanharo, ${ }^{4}$ Ruth Ester Assayag Batista ${ }^{5}$

'Enfermeira. Residente de Enfermagem. Universidade Federal De São Paulo. São Paulo-SP-Brasil. ${ }^{2}$ Enfermeira. Doutora em Enfermagem. Universidade Federal de São Paulo. São Paulo-SP-Brasil. ${ }^{3}$ Enfermeira. Mestre em Enfermagem. Universidade Federal de São Paulo. São Paulo-SP-Brasil.

${ }^{4}$ Enfermeira. Doutora em Ciências. Universidade Federal de São Paulo. São Paulo-SP-Brasil.

${ }^{5}$ Enfermeira. Doutora em Enfermagem. Professora da Universidade Federal de São Paulo. São Paulo-SP-Brasil.

RESUMO: O objetivo do estudo foi avaliar a fragilidade de idosos internados no Serviço de Emergência de um hospital universitário do estado de São Paulo. O período da coleta de dados foi de março a junho de 2014. Estudo transversal com 101 idosos, por período igual ou superior a 24 horas. Análise realizada pela aplicação da Edmonton Frail Scale. A média de idade foi 75 anos, 50,5\% sexo feminino, 58,4\% não terminou o ensino fundamental, 89,1\% aposentados ou pensionistas e 84,2\% acompanhados de cuidador. Antecedentes frequentes foram: hipertensão arterial $(65,3 \%)$, diabetes mellitus $(65,3 \%)$ e tabagismo $(44,6 \%)$. Em relação à fragilidade, a média do escore total foi 9,85, indicando fragilidade moderada. Os idosos com doenças neurológicas, demência, idade mais avançada e que tinham cuidador apresentaram maior fragilidade. O rastreamento da fragilidade propicia planejamento com vistas à prevenção de incapacidade e o agravamento das condições de saúde dos idosos. DESCRITORES: Idoso fragilizado; Idoso; Assistência à saúde.

\section{FRAILTY ASSESSMENT OF ELDERLY HOSPITALIZED AT AN EMERGENCY SERVICE OF A UNIVERSITY HOSPITAL}

\begin{abstract}
The objective in this study was to assess the frailty of elderly hospitalized at the Emergency Service of a university hospital in the State of São Paulo, Brazil. The data were collected between March and June 2014. Cross-sectional study involving 101 elderly, hospitalized for 24 hours or more. To develop the analysis, the Edmonton Frail Scale was applied. The mean age was 75 years, $50.5 \%$ female, $58.4 \%$ did not finish primary education, $89.1 \%$ retired or pensioner and $84.2 \%$ accompanied by a caregiver. Frequent antecedents were: arterial hypertension $(65.3 \%)$, diabetes mellitus (65.3\%) and smoking (44.6\%). Concerning the frailty, the mean total score was 9.85, indicating moderate frailty. The elderly people with neurological diseases, dementia, more advanced age and who had a caregiver demonstrated higher levels of frailty. Frailty screening favors planning with a view to preventing disability and the worsening of the elderly people's health conditions.
\end{abstract}

DESCRIPTORS: Frail elderly; Aged; Delivery of health care.

\section{EVALUACIÓN DE LA FRAGILIDAD DE ANCIANOS INTERNADOS EN SERVICIO DE EMERGENCIA DE UN HOSPITAL UNIVERSITARIO*}

RESUMEN: El objetivo del estudio fue evaluar la fragilidad de ancianos internados en el Servicio de Emergencia de un hospital universitario del estado de São Paulo. El periodo en que los datos fueron obtenidos fue de marzo a junio de 2014. Estudio transversal con 101 ancianos, por periodo igual o superior a 24 horas. Análisis realizado por aplicación de la Edmonton Frail Scale. La media de edad fue 75 años, 50,5\% sexo femenino, 58,4\% no completó la enseñanza fundamental, $89,1 \%$ jubilados o pensionistas y $84,2 \%$ acompañados de cuidador. Antecedentes frecuentes fueron: hipertensión arterial $(65,3 \%)$, diabetes mellitus $(65,3 \%)$ y tabaquismo $(44,6 \%)$. Acerca de la fragilidad, la media del score total fue 9,85, apuntando fragilidad moderada. Los ancianos con enfermedades neurológicas, demencia, edad más avanzada y que tenían cuidador presentaron más fragilidad. Rastrear la fragilidad propicia planeamiento para prevenir la incapacidad y el agravamiento de las condiciones de salud de los ancianos. DESCRIPTORES: Anciano fragilizado; Anciano; Asistencia a la salud.

*Artigo extraído do trabalho de conclusão de curso de graduação em Enfermagem intitulado: "Interação medicamentosa nas prescrições médicas de idosos internados no Serviço de Emergência de um Hospital Universitário". Escola Paulista de Enfermagem da Universidade Federal de São Paulo, 2014.

Autor Correspondente:

Cássia Regina Vancini Campanharo

Universidade Federal de São Paulo

Rua Napoleão de Barros, 754 - 04024-002 - São Paulo-SP-Brasil

E-mail: cvancini@unifesp.br
Recebido: $19 / 02 / 2015$

Finalizado: 10/05/2015 


\section{INTRODUÇÃO}

De acordo com recentes dados divulgados das Nações Unidas, nas regiões mais desenvolvidas a população acima de 60 anos tem perspectiva de aumentar em $45 \%$ até o meio do século, passando de 287 milhões em 2013 para 417 milhões em 2050. Em regiões menos desenvolvidas, esta população também terá crescimento significativo, espera-se que ela aumente de 554 milhões em 2013 para 1.6 bilhões em 2050 ${ }^{(1)}$.

O Instituto Brasileiro de Geografia e Estatística (IBGE), em 2010, demonstrou que o Brasil está passando por uma transição demográfica, na qual os idosos representam aproximadamente 10,8\% da população total do país. A expectativa para o ano de 2050, é que existirão mais idosos que crianças abaixo de 15 anos de idade ${ }^{(2)}$.

A prevalência de doenças crônicas não transmissíveis acompanha o aumento da expectativa de vida. Além disso, as doenças crônicas podem comprometer a capacidade funcional do idoso, acelerando o processo da síndrome da fragilidade ${ }^{(3)}$.

A síndrome da fragilidade é multifatorial e envolve fatores biológicos, físicos, cognitivos, sociais, econômicos e ambientais e resulta do declínio cumulativo dos sistemas. A fragilidade torna o idoso mais dependente para suas atividades de vida diária (AVDs), podendo acarretar exaustão, fraqueza, incontinência urinária, distúrbio do equilíbrio, marcha vagarosa e diminuição da atividade física, sendo potencial causa de quedas, hospitalização, institucionalização, declínio funcional e morte ${ }^{(4-5)}$.

Os Serviços de Emergência (SE) são unidades com frequente superlotação de pacientes, insuficiência de recursos humanos e físicos. Referente às especificidades do cuidado, estes serviços requerem equipe especializada para o atendimento, de maneira rápida, à pacientes em estado crítico. Ainda, cabe à equipe, resguardar a segurança do paciente e familiar, bem como oferecer apoio emocional para ambos. Nesses locais faz-se necessária uma adaptação ao novo perfil epidemiológico da população ${ }^{(6)}$.

Considerando a dificuldade para estabelecer conceitos relacionados à definição da fragilidade em idosos, é fundamental a aplicação de instrumentos confiáveis que possam ajudar na identificação de idosos frágeis e potencialmente frágeis para a individualização da assistência. No Brasil, a Edmonton Frail Scale (EFS) é utilizada por profissionais da saúde para a detecção de fatores de risco para fragilidade, determinação do nível de fragilidade do idoso e dos principais domínios que necessitam de intervenção, o que pode prevenir seu avanço, reduzindo o tempo de internação e melhorando a qualidade de vida desses indivíduos ${ }^{(7)}$.

Diante do atual crescimento da população idosa brasileira, da prevalência das doenças crônicas e do aumento da procura por parte desta população pelos SE, este estudo tem o objetivo de avaliar a fragilidade de idosos internados em uma unidade com esta característica, para a detecção precoce e planejamento da assistência às necessidades desta população.

\section{MÉTODO}

Estudo transversal realizado em Serviço de Emergência de um hospital universitário, localizado no município de São Paulo, aprovado pelo Comitê de Ética em Pesquisa da Universidade Federal de São Paulo (CEP: 631956; CAAE: 28238814.8.0000.5505). Os participantes foram incluídos no estudo após leitura e assinatura do termo de consentimento livre e esclarecido.

Os critérios de inclusão foram indivíduos a partir de 60 anos de idade ${ }^{(8)}$, internados no SE durante o período de coleta de dados, março a junho de 2014, totalizando em uma amostra aleatória de 101 pacientes.

A avaliação da fragilidade foi realizada por meio da aplicação da EFS e realizada por uma das pesquisadoras, por meio de entrevista com o idoso, familiar ou cuidador, no período da tarde, das 14 às 17 horas, em dias alternados. Foram pesquisadas as variáveis: gênero, idade, cor da pele, escolaridade, emprego, renda familiar, presença de cuidador, comorbidades, hábitos e diagnóstico médico na internação por meio da entrevista ou busca no prontuário do paciente.

A EFS é composta por nove domínios: Cognição (0-2 pontos); Estado Geral de Saúde (04); Independência Funcional (0-2); Suporte Social (0-2); Uso de Medicamentos (0-2); Nutrição (0-1); Humor (0-1); Continência (0-1) e Desempenho Funcional $(0-2)^{(7)}$. Os escores da escala são: 0 a 4 
não apresenta fragilidade; 5 a 6 é aparentemente frágil; 7 a 8 fragilidade leve; 9 a 10 fragilidade moderada e de 11 ou mais pontos a fragilidade é severa. Quanto maior a pontuação da EFS maior o nível de fragilidade do idoso ${ }^{(9)}$.

Para comparar os domínios da EFS com a idade e o número de dependentes utilizou-se a Análise de Variância (ANOVA) e com as variáveis categóricas o teste Qui-Quadrado e, quando necessário, a Razão de Verossimilhança ou Exato de Fisher. Para comparar o escore total da escala com a idade e número de dependentes utilizou-se o coeficiente de correlação de Spearman e com as variáveis categóricas a Análise de Variância (ANOVA). O nível de significância utilizado foi $5 \%(p$-valor $\leq 0,05)$.

\section{RESULTADOS}

A média de idade dos pacientes foi 75 anos, $50,5 \%(n=51)$ do sexo feminino, 58,4\% que não terminaram o ensino fundamental, $89,1 \%$ declararam-se aposentados ou pensionistas e $84,2 \%$ estavam acompanhados de cuidador. A hipertensão arterial (65,3\%), o diabetes mellitus $(65,3 \%)$ e tabagismo $(44,6 \%)$ foram os antecedentes mais frequentes nesta população. O escore de fragilidade dos idosos neste estudo variou de 2 a 16.

A Tabela 1 apresenta o escore total de fragilidade e a fragilidade por domínios da EFS dos pacientes internados no Serviço de Emergência.

Como demonstrado na Tabela 2 os idosos com cuidador apresentaram maior percentual de reprovação com erros significativos no teste do desenho de um relógio (Domínio Cognição), maior percentual de internação, dependência funcional, perda de peso, tristeza ou depressão e levaram mais que 20 segundos para completar o teste do "Levante e ande" quando comparados aos idosos que não tinham cuidador.

Pacientes com baixa escolaridade levaram mais que 20 segundos para completar o teste "Levante e ande" quando comparados aos com maior escolaridade; e aqueles com emprego consideraram sua saúde ruim e levaram até 10 segundos para completar o teste "Levante e ande" quando comparados àqueles que não tinham emprego (Tabela 2).

Pacientes com maior escolaridade
Tabela 1 - Avaliação da fragilidade por meio da Edmonton Frail Scale dos idosos internados em Serviço de Emergência ( $n=101)$. São Paulo-SP-Brasil, 2014

\begin{tabular}{|c|c|}
\hline Domínios da Edmonton Frail Scale & $\%$ \\
\hline \multicolumn{2}{|l|}{ Nutrição (perda de peso) } \\
\hline Sim & 77,2 \\
\hline Não & 22,8 \\
\hline \multicolumn{2}{|c|}{ Esquecimento do uso de medicamentos } \\
\hline Sim & 44,6 \\
\hline Não & 55,4 \\
\hline \multicolumn{2}{|l|}{ Suporte Social } \\
\hline Sempre & 85,1 \\
\hline Algumas vezes & 13,9 \\
\hline Nunca & 1 \\
\hline \multicolumn{2}{|l|}{ Independência Funcional } \\
\hline Até 1 atividade & 15,8 \\
\hline De 2 a 4 atividades & 22,8 \\
\hline De 5 a 8 atividades & 61,4 \\
\hline \multicolumn{2}{|l|}{ Cognição } \\
\hline Aprovado & 4,4 \\
\hline Reprovado com erros mínimos & 3,3 \\
\hline Reprovado com erros significantes & 92,3 \\
\hline \multicolumn{2}{|l|}{ Usa cinco ou mais medicamentos } \\
\hline Sim & 53,5 \\
\hline Não & 46,5 \\
\hline \multicolumn{2}{|l|}{ Incontinência } \\
\hline Sim & 59,4 \\
\hline Não & 40,6 \\
\hline \multicolumn{2}{|l|}{ Humor (triste/deprimido) } \\
\hline Sim & 55,4 \\
\hline Não & 44,6 \\
\hline \multicolumn{2}{|c|}{ Desempenho funcional (teste "Levante e ande") } \\
\hline Até 10 segundos & 5,9 \\
\hline De 11 a 20 segundos & 11,9 \\
\hline Mais que 20 segundos & 82,2 \\
\hline \multicolumn{2}{|c|}{ Estado Geral de Saúde (descrição da saúde) } \\
\hline Excelente/Muito boa/Boa & 14,9 \\
\hline Razoável & 57,4 \\
\hline Ruim & 27,7 \\
\hline \multicolumn{2}{|l|}{ Estado Geral de Saúde (internação) } \\
\hline Nenhuma vez & 40,6 \\
\hline De 1 a 2 vezes & 38,6 \\
\hline Mais que 2 vezes & 20,8 \\
\hline \multicolumn{2}{|l|}{ Escore total } \\
\hline Média (DP) & $9,85(3,0)$ \\
\hline Mediana (mínimo-máximo) & $10(2-16)$ \\
\hline
\end{tabular}


Tabela 2 - Relação entre as variáveis emprego e presença de cuidador e os Domínios da Edmonton Frail Scale, nos idosos internados em Serviço de Emergência (n=101). São Paulo, SP, Brasil, 2014

\begin{tabular}{|c|c|c|c|c|}
\hline \multirow{2}{*}{ Domínios da Edmonton Frail Scale } & \multicolumn{2}{|c|}{ Presença de cuidador $(\%)$} & \multicolumn{2}{|c|}{ Emprego (\%) } \\
\hline & Sim & Não & Sim & Não \\
\hline \multicolumn{5}{|l|}{ Cognição } \\
\hline Aprovado & $3,9^{*}$ & 7,1 & $-* * *$ & 4,7 \\
\hline Reprovado com erros mínimos & - & 21,4 & - & 3,5 \\
\hline Reprovado com erros significantes & 96,1 & 71,4 & 100 & 91,9 \\
\hline \multicolumn{5}{|l|}{ EGS (internações no último ano) } \\
\hline Nenhuma vez & $35,3^{* *}$ & 68,8 & $20^{* * *}$ & 41,7 \\
\hline De 1 a 2 vezes & 41,2 & 25 & 80 & 36,5 \\
\hline Mais que 2 vezes & 23,5 & 6,3 & - & 21,9 \\
\hline \multicolumn{5}{|l|}{ EGS } \\
\hline Excelente/Muito boa/ Boa & $8,2^{*}$ & 56,3 & $40^{* * *}$ & 14,6 \\
\hline Razoável & 23,5 & 18,8 & - & 24 \\
\hline Ruim & 68,2 & 25 & 60 & 61,5 \\
\hline \multicolumn{5}{|l|}{ Independência Funcional } \\
\hline Até 1 atividade & $8,2^{*}$ & 56,3 & $40^{* * *}$ & 14,6 \\
\hline De 2 a 4 atividades & 23,5 & 18,8 & - & 24 \\
\hline De 5 a 8 atividades & 68,2 & 25 & 60 & 61,5 \\
\hline \multicolumn{5}{|l|}{ Suporte social } \\
\hline Sempre & $89,4^{* * *}$ & 62,5 & $100^{* * *}$ & 84,4 \\
\hline Algumas vezes & 10,6 & 31,3 & - & 14,6 \\
\hline Nunca & - & 6,3 & - & 1 \\
\hline \multicolumn{5}{|l|}{ Usa cinco ou mais medicamentos } \\
\hline Sim & $55,3^{* * *}$ & 43,8 & $40^{* * *}$ & 54,2 \\
\hline Não & 44,7 & 56,3 & 60 & 45,8 \\
\hline \multicolumn{5}{|l|}{ Esquece-se de tomar o remédio } \\
\hline Sim & 44,7 & 43,8 & $60^{* * *}$ & 43,8 \\
\hline Não & 55,3 & 56,3 & 40 & 56,3 \\
\hline \multicolumn{5}{|l|}{ Nutrição } \\
\hline Sim & $82,4^{*}$ & 50 & $60^{* * *}$ & 78,1 \\
\hline Não & 17,6 & 50 & 40 & 21,9 \\
\hline \multicolumn{5}{|l|}{ Humor (triste/deprimido) } \\
\hline Sim & $61,2^{*}$ & 25 & - & 57,3 \\
\hline Não & 38,8 & 75 & 80 & 42,7 \\
\hline \multicolumn{5}{|l|}{ Incontinência } \\
\hline Sim & $63,5^{* * *}$ & 37,5 & $20^{* * *}$ & 61,5 \\
\hline Não & 36,5 & 62,5 & 80 & 38,5 \\
\hline \multicolumn{5}{|l|}{ Desempenho funcional } \\
\hline Até 10 segundos & $2,4^{* *}$ & 25 & $40^{* *}$ & 4,2 \\
\hline De 11 a 20 segundos & 11,8 & 12,5 & - & 12,5 \\
\hline Mais que 20 segundos & 85,9 & 62,5 & 60 & 83,3 \\
\hline
\end{tabular}

Teste Qui-quadrado, quando necessário o teste da Razão de Verossimilhança ou Exato de Fisher. *Valor de $p \leq 0,01$;**Valor de $p$ $\leq 0,05 ; * * *$ Valor de p >0,05; EGS- Estado Geral de Saúde. 
apresentaram maior independência funcional $(p=0,0334)$ e esqueceram-se mais de tomar seus medicamentos quando comparados aqueles com menor escolaridade ( $p=0,0017)$.

Os pacientes que consideraram sua saúde ruim tiveram maior número de dependentes da renda quando comparados àqueles que consideraram sua saúde excelente, muito boa ou boa ( $p=0,0471)$. $E$ idosos com incontinência apresentaram maior idade $(p<0,0001)$.

As comorbidades dos idosos que tiveram associação com domínios da EFS foram doenças neurológicas, demência, cardiopatia, doença osteomuscular e diabetes mellitus.

Os idosos com doenças neurológicas consideraram sua saúde ruim $(p=0,0162)$. Os pacientes com doença neurológica $(p=0,0421)$ e demência $(p=0,0001)$ apresentaram maior percentual de dependência funcional. Aqueles com demência apresentaram maior perda de peso $(p=0,0141)$ e relataram se sentir mais tristes ou deprimidos $(p=0,0144)$ e apresentaram maior percentual de incontinência quando comparados com aqueles com outras comorbidades ( $p=0,0014)$. Os pacientes com demência $(p=0,0249)$ e doença osteomuscular $(p=0,0317)$ levaram até 10 a 20 segundos para completar o teste de desempenho funcional quando comparados àqueles com outras comorbidades. Os idosos com diabetes mellitus $(p=0,0307)$, cardiopatia $(p=0,0250)$ e doença osteomuscular $(p=0,0151)$ faziam usos de cinco ou mais medicamentos prescritos pelo médico.

Os idosos com doenças neurológicas, demência e aqueles que tinham cuidador apresentaram maior nível de fragilidade. Além disso, aqueles com mais idade também apresentaram maior pontuação na EFS ( $p=$ 0,0266) (Tabela 3).

Tabela 3 - Relação entre o escore total da Edmonton Frail Scale e as variáveis: presença de cuidador, doenças neurológicas e demência dos idosos internados em Serviço de Emergência (n=101). São Paulo-SP-Brasil, 2014

\begin{tabular}{|c|c|c|c|c|c|}
\hline & \multicolumn{5}{|c|}{ Total de Pontos da Edmonton Frail Scale } \\
\hline & $\mathrm{n}$ & Média (DP) & Mediana & Mínimo-Máximo & p-valor* \\
\hline \multicolumn{6}{|c|}{ Presença de Cuidador } \\
\hline Sim & 85 & $10,3(2,7)$ & 11 & $3-16$ & \multirow{2}{*}{$<0,0001$} \\
\hline Não & 16 & $7,1(2,9)$ & 7 & $2-14$ & \\
\hline \multicolumn{6}{|c|}{ Doenças neurológicas } \\
\hline Sim & 16 & $11,6(2,2)$ & 12 & $7-16$ & \multirow{2}{*}{0,0071} \\
\hline Não & 85 & $9,5(3)$ & 10 & $2-15$ & \\
\hline \multicolumn{6}{|c|}{ Demência } \\
\hline Sim & 17 & $12,2(2,1)$ & 12 & $8-16$ & \multirow{2}{*}{0,0002} \\
\hline Não & 84 & $9,3(2,9)$ & 10 & $2-14$ & \\
\hline
\end{tabular}

*Análise de Variância (ANOVA). Nível de significância de 5\% (p-valor < 0,05).

\section{DISCUSSÃO}

O escore de fragilidade dos idosos deste estudo foi semelhante a outro realizado com pacientes internados em unidade de clínica médica $(6$ a 16 pontos)(10). Além disso, os pacientes desta pesquisa apresentaram comprometimento de 7 dos 9 domínios da EFS, o que diverge do estudo realizado em Unidade de Saúde da Família, no qual houve comprometimento apenas na cognição(11). Este resultado pode estar relacionado ao fato desses idosos não estarem internados e não apresentarem comprometimento agudo.
A maior frequência de reprovação no domínio cognição, dependência funcional e tristeza ou depressão dos idosos com cuidador provavelmente são fatores que contribuem para maior dependência para realização das AVDs e maior necessidade de cuidador ${ }^{(12)}$. Foi observado em um estudo, que quanto maior a dependência funcional do idoso, ou seja, quanto menor sua capacidade para realizar atividades do cotidiano, preservar suas atividades mentais e sociais, maior a necessidade de um cuidador ${ }^{(13)}$.

Pacientes com baixa escolaridade levaram mais que 20 segundos para completar o teste de desempenho funcional quando comparados aos 
com maior escolaridade; e aqueles com emprego consideraram sua saúde ruim e levaram até 10 segundos para completar o teste de desempenho funcional. A incidência de fragilidade em idosos está relacionada ao baixo nível socioeconômico e à pouca escolaridade, por afetar, especialmente, seu estilo de vida e aumentar sua exposição a riscos para a saúde, além de potencializar a condição física da fragilidade ${ }^{(9)}$.

O trabalho deve estar adaptado ao trabalhador, sua ação sobre este pode potencializar sua saúde, porém dependendo da organização do processo laboral, poderá representar risco à saúde e ser causa de doenças profissionais ou agravar um estado de saúde deficiente de origem extraprofissional ${ }^{(14)}$. Isto pode explicar porque aqueles idosos com emprego consideraram sua saúde ruim.

Pacientes com mais escolaridade apresentaram maior independência funcional, semelhante aos resultados observados em outro estudo ${ }^{(13)}$, em que os idosos com baixa escolaridade possuíam até cinco vezes maior chance de apresentar dependência de moderada a grave. Nesse estudo os pacientes com maior escolaridade foram aqueles que não se lembraram de tomar seus medicamentos, em contradição com uma pesquisa, que apesar do achado não ter significância estatística, os indivíduos com menor escolaridade tiveram menor adesão ao tratamento $^{(15)}$.

Neste estudo os pacientes que consideram sua saúde como ruim possuem maior número de dependentes da renda quando comparados àqueles que consideram sua saúde excelente, muito boa ou boa. Muitos idosos contribuem para o orçamento familiar, tornando a aposentadoria uma garantia financeira para filhos desempregados e netos; e quando esta renda não é suficiente para realizar as necessidades tanto dos idosos quanto de suas famílias, pode expor os idosos ao risco de adoecimento ou até agravar suas doenças existentes ${ }^{(16-17)}$.

Os idosos com doenças neurológicas consideraram sua saúde ruim. Os pacientes com doença neurológica e demência apresentaram maior percentual de dependência funcional; e aqueles com demência apresentaram maior perda de peso além de relatarem se sentir mais tristes ou deprimidos e apresentarem maior percentual de incontinência urinária quando comparados com aqueles com outras comorbidades. A maior dependência para as AVDs e o comprometimento do controle do esfíncter urinário dos idosos com demência ou com doença neurológica também foram constatados em outro estudo ${ }^{(14)}$.

Os idosos com incontinência urinária nesta pesquisa foram aqueles com maior idade. Embora o trato urinário inferior sofra mudanças associadas ao envelhecimento tanto no homem quanto na mulher ${ }^{(18)}$, a incontinência pode afetar pessoas de diferentes idades, e estar associada ao maior risco de declínio funcional ${ }^{(19)}$.

Alguns fatores de risco para transtornos depressivos foram observados nos idosos entrevistados, como idade avançada, ansiedade, doenças crônicas, a falta de vínculos e suporte social( $^{(20)}$. A depressão é uma condição clínica muito importante nos idosos, já que abala de forma negativa a capacidade funcional, sua qualidade de vida, além de afetar sua autonomia colaborando com a evolução de sua fragilidade ${ }^{(21)}$.

Os pacientes com demência e doença osteomuscular levaram de 10 a 20 segundos para completar o teste de desempenho funcional quando comparados àqueles com outras comorbidades. Semelhante ao resultado deste estudo, pesquisa em instituição de longa permanência identificou que idosos com demência tinham maior comprometimento do desempenho funcional. As doenças osteosmuculares também causaram dificuldade de mobilidade, inclusive para realização de atividades como uso de escadas ou tomar banho ${ }^{(14)}$.

Os idosos com diabetes mellitus, cardiopatia e doenças osteomusculares faziam uso de cinco ou mais medicamentos prescritos pelo médico. A ocorrência da polifarmácia acontece também graças ao aumento das doenças crônicas não transmissíveis. Estudo que avaliou o nível de fragilidade em 63 idosos participantes de um centro de convivência demonstrou que quanto maior o número de medicações maior o escore de fragilidade ${ }^{(5)}$.

Os idosos com doenças neurológicas, demência e aqueles que tinham cuidador apresentaram maior escore total na EFS, ou seja, maior o nível de fragilidade. Doenças neurológicas como Alzheimer, causam comprometimento da capacidade funcional, assim os idosos precisam de cuidadores para o auxílio de suas atividades diárias. Já a demência é uma síndrome, com 
declínio gradual da função cognitiva, com mudança de personalidade, comprometimento para realização das atividades de vida diária, fazendo com que o idoso torne-se cada vez mais dependente e fragilizado ${ }^{(18)}$.

Os pacientes com mais idade apresentaram maior pontuação na EFS, sendo estes os mais fragilizados. Isto sugere que quanto maior a idade, maior a tendência à fragilidade ${ }^{(22)}$.

\section{CONCLUSÃO}

Os idosos tiveram fragilidade moderada, aqueles com cuidador apresentaram maior percentual de internação, maior dependência, perda de peso, tristeza ou depressão. Pacientes com baixa escolaridade levaram mais tempo para completar o teste de desempenho funcional; e aqueles com mais escolaridade apresentaram maior independência funcional. As doenças neurológicas, demência, cardiopatia, doença osteomuscular e diabetes mellitus tiveram associação com a EFS. Os idosos com doenças neurológicas, demência, os que tinham cuidador e mais idade apresentaram o maior nível de fragilidade.

Os resultados deste estudo podem contribuir para inserir medidas de rastreamento da fragilidade entre os idosos, de modo a identificar e corrigir precocemente esses fatores para prevenir ou minimizar a ocorrência de eventos indesejáveis, como a incapacidade e o agravamento das condições de saúde dos idosos.

A principal limitação deste estudo é o fato de ter sido realizado em um único hospital, que presta assistência à pacientes do sistema público e pode não representar outras realidades.

\section{REFERÊNCIAS}

1. United Nations, Department of Economic and Social Affairs, Population Division. World population prospects: the 2012 revision, key findings and advance tables. New York, 2013 [acesso em 05 mai 2015]. Disponível: http://esa.un.org/wpp/documentation/pdf/ wpp2012_\%20key\%20findings.pdf

2. Instituto Brasileiro de Geografia e Estatística (IBGE). Sinopse do censo demográfico 2010. Sinopse do censo e resultados preliminares do universo. [acesso em 01 abr 2015]. Disponível: http://www.ibge.gov.br/home/ presidencia/noticias/imprensa/ppts/0000000402.pdf

3. Kuchemann BA. Envelhecimento populacional, cuidado e cidadania: velhos dilemas e novos desafios. Soc estado. 2012;27(1):165-80.

4. Nogueira SL, Ribeiro RCL, Rosado LEFPL, Franceschini SCC, Ribeiro AQ, Pereira ET. Fatores determinantes da capacidade funcional em idosos longevos. Rev Bras Fisioterapia. 2010;14(4):322-9.

5. Carmo LV, Drummond LP, Arantes PMM. Avaliação do nível de fragilidade em idosos participantes de um grupo de convivência. Fisioter Pesqui. 2011;18(1):17-22.

6. Ministério da Saúde (BR). Secretaria de Atenção à Saúde. Departamento de Atenção Básica. Envelhecimento e saúde da pessoa idosa. Cadernos de Atenção Básica № 19. Brasília (DF), 2006.

7. Fabrício-Wehbe SCC, Shciaveto FV, Vendrusculo TRP, Haas VJ, Dantas RAS, Rodrigues RAP. Adaptação cultural e validade da Edmont Frail Scale - EFS em uma amostra de idosos brasileiros. Rev Latino-Am. Enfermagem. 2009,17(6):1043-9.

8. Brasil. Lei $n^{\circ}$. 8.842. Dispõe sobre a política nacional do idoso, cria o Conselho Nacional do Idoso e dá outras providências. [acesso em 01 abr 2015]. Disponível: http://www.planalto.gov.br/ccivil_03/leis/ I8842.htm

9. Ferreira PCS, Tavares DMS, Rodrigues RAP. Características sociodemográficas, capacidade funcional e morbidades entre idosos com e sem declínio cognitivo. Acta paul enferm. 2011;24(1):29-35.

10. Storti LB, Fabrício-Whebe SCC, Kusumota L, Rodrigues RAP, Marques S. Fragilidade de idosos internados na clínica médica da unidade de emergência de um hospital geral terciário. Texto contexto enferm. 2013;22(2):452-9.

11. Maeshiro FL, Lopes MC, Okuno MF, Campanharo CR, Batista RE. Capacidade funcional e a gravidade do trauma em idosos. Acta paul enferm. 2013;26(4):38994.

12. Reis LA, Torres GV, Novaes LKN, Reis LA. Déficit cognitivo como fator de risco para a limitação de atividades cotidianas em idosos institucionalizados. Revista de Psicologia. 2011;2(1):126-36.

13. Talmelli LFS, Gratão ACM, Kusumota L, Rodrigues RAP. Nível de independência funcional e déficit cognitivo em idosos com Doença de Alzheimer. Rev Esc Enferm. USP. 2010;44(4):933-9.

14. Dantas CMHL, Bello FA, Barreto KL, Lima LS. Capacidade funcional de idosos com doenças crônicas residentes em Instituições de Longa Permanência. Rev bras enferm. 2013;66(6):914-20. 
15. Schmitt-Júnior AA, Lindner S, Helena ETS. Avaliação da adesão terapêutica em idosos atendidos na atenção primária. Rev. Assoc. Med. Bras. 2013;59(6):614-21.

16. Sá CMS, Souza NVDO, Caldas CP, Lisboa MTL, Tavares KFA. O idoso no mundo do trabalho: configurações atuais. Cogitare enferm. 2011;16(3):536-42.

17. Alvarenga MRM, Oliveira MAC, Domingues MAR, Amendola F, Faccenda O. Rede de suporte social do idoso atendido por equipes de saúde da família. Cienc. saude colet. 2011;16(5):2603-11.

18. Reis RB, Cologna AJ, Martins ACP, Paschoalin EL, Tucci Júnior S, Suaid HJ. Incontinência urinária no Idoso. Acta Cir. Bras. 2003;18(5):47-51.

19. Pavarini SCl, Melo LC, Silva VM, Orlandi FS, Mendiondo MSZ, Filizola CLA, et al. Cuidando de idosos com Alzheimer: a vivência de cuidadores familiares. Rev. Eletr. Enf. 2008;10(3):580-90.

20. Mitchell AJ, de Santiago AI. Prognosis of depression in the eldery in comparison with adult age. Is there a significant clinical difference? Actas Esp Psiquiatr. 2009;37(5):289-96.

21. Paradela EMP. Depressão em Idosos. Rev HUPE. 2011;10(2):31-40.

22. Duarte MCS, Fernandes MGM, Rodrigues RAP, Nóbrega MML. Prevalência e fatores sociodemográficos associados à fragilidade em mulheres idosas. Rev bras enferm. 2013;66(6):901-6. 